OPEN ACCESS

Edited by:

Maria Helena S. Goldman, University of São Paulo, Brazil

Reviewed by:

Emily Bellis,

Arkansas State University,

United States

Lynette Ruth Brownfield,

University of Otago, New Zealand

*Correspondence:

Ki-Hong Jung

khjung2010@khu.ac.kr

Yu-Jin Kim

yjkim2020@pusan.ac.kr

Specialty section:

This article was submitted to Plant Development and EvoDevo,

a section of the journal

Frontiers in Plant Science

Received: 05 November 2020

Accepted: 22 March 2021

Published: 13 April 2021

Citation:

Lee SK, Hong W-J, Silva J,

Kim E-J, Park SK, Jung K-H and Kim Y-J (2021) Global Identification

of ANTH Genes Involved in Rice

Pollen Germination and Functional Characterization of a Key Member,

OSANTH3.

Front. Plant Sci. 12:609473. doi: 10.3389/fp/s.2021.609473

\section{Global Identification of ANTH Genes Involved in Rice Pollen Germination and Functional Characterization of a Key Member, OsANTH3}

\author{
Su Kyoung Lee1, Woo-Jong Hong', Jeniffer Silva1, Eui-Jung Kim¹, Soon Ki Park², \\ Ki-Hong Jung ${ }^{1 *}$ and Yu-Jin Kim ${ }^{3 *}$
}

${ }^{1}$ Graduate School of Biotechnology, Crop Biotech Institute, Kyung Hee University, Yongin, South Korea, ${ }^{2}$ School of Applied Biosciences, Kyungpook National University, Daegu, South Korea, ${ }^{3}$ Department of Life Science and Environmental Biochemistry, Life and Industry Convergence Research Institute, Pusan National University, Miryang, South Korea

Pollen in angiosperms plays a critical role in double fertilization by germinating and elongating pollen tubes rapidly in one direction to deliver sperm. In this process, the secretory vesicles deliver cell wall and plasma membrane materials, and excessive materials are sequestered via endocytosis. However, endocytosis in plants is poorly understood. AP180 N-terminal homology (ANTH) domain-containing proteins function as adaptive regulators for clathrin-mediated endocytosis in eukaryotic systems. Here, we identified 17 ANTH domain-containing proteins from rice based on a genomewide investigation. Motif and phylogenomic analyses revealed seven asparagineproline-phenylalanine (NPF)-rich and 10 NPF-less subgroups of these proteins, as well as various clathrin-mediated endocytosis-related motifs in their C-terminals. To investigate their roles in pollen germination, we performed meta-expression analysis of all genes encoding ANTH domain-containing proteins in Oryza sativa (OsANTH genes) in anatomical samples, including pollen, and identified five mature pollenpreferred OsANTH genes. The subcellular localization of four OsANTH proteins that were preferentially expressed in mature pollen can be consistent with their role in endocytosis in the plasma membrane. Of them, OsANTH3 represented the highest expression in mature pollen. Functional characterization of OSANTH3 using T-DNA insertional knockout and gene-edited mutants revealed that a mutation in OSANTH3 decreased seed fertility by reducing the pollen germination percentage in rice. Thus, our study suggests OsANTH3-mediated endocytosis is important for rice pollen germination.

Keywords: AP180 N-terminal homology protein, endocytosis, gene editing (CRISPR-Cas9), Oryza sativa, pollen germination

\section{INTRODUCTION}

Like all eukaryotes, plant cells are composed of intracellular compartments with proteins located either inside or in the plasma membrane (PM). Thus, the precise transport of proteins and localization of certain proteins are critical for the normal function of cells, requiring complex and systematic regulation of membrane trafficking (Zouhar and Sauer, 2014). The balanced processes 
of endocytosis and exocytosis/secretion maintain proteins and lipids for the PM in addition to regulating transport and signaling. Endocytosis is defined as the uptake of membraneassociated and soluble cargo from the extracellular space through the formation of a vesicle at the PM. Due to its applications in human health, endocytosis has been extensively studied in yeast and mammalian cell systems, compared with limited recent investigations in plants (Robinson et al., 2008; Reynolds et al., 2018).

When pollen grains land on the stigma in higher plants, compatible grains germinate, and an elongating pollen tube forms and grows into the style toward the ovules. Germination of pollen grains is a complex biological event that requires numerous factors and activities (Wang et al., 2010). Many of these factors, such as nutrients, proteins, signaling molecules, and various cell components, are contained in vesicles. The vesicles carrying cargo molecules are involved in several stages of endocytic pathways, of which clathrinmediated endocytosis (CME) is one of the major endocytic activities (Lacy et al., 2018). The process of pollen germination is essential for successful reproduction in plants; however, little is known regarding the mechanism of selective internalization or molecular sorting during pollen germination.

Endocytosis processes include various steps, such as cargo collection, vesicle assembly and invagination from the PM, and trafficking to the trans-Golgi network (TGN)/early endosome (EE), that are mediated by coat proteins as well as adaptor and accessory proteins (Nguyen et al., 2018). Clathrin, which functions as a coat protein during vesicle formation, does not directly interact with cargoes; instead, it interacts via adaptor proteins. The epsin N-terminal homology (ENTH) domain acts as an adaptor during CME in animal cells. Proteins with a similar domain, the AP180 N-terminal homology (ANTH) domain, also function in clathrin-coated vesicle (CCV) formation and cargo sorting at the PM during CME (Duncan and Payne, 2003; Legendre-Guillemin et al., 2004). In ANTH domain-containing proteins (from here on referred to as ANTH proteins encoded by $A N T H$ genes), the N-terminus of the ANTH domain is located in the PM and possess affinity to phosphatidylinositol 4,5biphosphate, and the C-terminus is involved in CCV formation in combination with other elements such as clathrin (Itoh et al., 2001; Zouhar and Sauer, 2014). The yeast AP180 protein functions at the budding region of the PM (Legendre-Guillemin et al., 2004), and in neurons the AP180 proteins are enriched in the presynaptic terminal membrane (Yao et al., 2002), suggesting their association with polar growth.

Plants contain a larger number of ANTH genes than mammals or yeast; however, only a few have been shown to function in cargo trafficking (Barth and Holstein, 2004; Holstein and Oliviusson, 2005; Zouhar and Sauer, 2014). AtAP180/PICALM6 has been extensively shown to interact with clathrin and accelerate cargo efficiency in vitro (Barth and Holstein, 2004). Arabidopsis epsin-like clathrin adaptor (AtECA) protein 1, AtECA2/PICALM5a, and PICALM4 have shown involvement in cytokinesis during cell plate expansion (Song et al., 2012; Nguyen et al., 2018). Recent studies demonstrated the role of ANTH domain-containing proteins in Arabidopsis pollen tube growth, such as acting as adaptors of CCV or engaging in pollen tube PM integrity (Muro et al., 2018; Kaneda et al., 2019). PICALM5b and AtECA2/PICALM5a are involved in maintaining pollen tube integrity and tip localization of receptor kinases during CME (Muro et al., 2018). Endocytosis adaptor of pollen tube 1 (EAP1) interacts with REN4 (WD40 domain protein) and REN4 interacting partner 1 (ROP1) and then removes both proteins from the PM by CME to maintain polar tip growth of the pollen tube (Li et al., 2018).

The role or even the existence of the ANTH gene family in monocot plants is not well understood. In this study, we investigated the genome-wide characteristics of ANTH domaincontaining proteins in Oryza sativa (rice), named OsANTH, and performed domain and phylogenetic analyses with Arabidopsis ANTH proteins. The highest expressed gene in rice pollen, OsANTH3, was further analyzed through characterization of the T-DNA insertional knockout mutant and gene-edited mutants. These findings are expected to contribute to a comprehensive understanding of the ANTH family in rice and their role in late pollen development.

\section{MATERIALS AND METHODS}

\section{Plant Materials and Growth Conditions}

Two japonica rice (Oryza sativa subsp. japonica) varieties, Dongjin (DJ) and Nipponbare (NP), as the wild-type (WT) plants, together with the T-DNA insertional mutants in the DJ background were grown in either a growth chamber under a day/night cycle of $28^{\circ} \mathrm{C}$ ( $16 \mathrm{~h}$ light) and $22^{\circ} \mathrm{C}$ ( $8 \mathrm{~h}$ dark) with $\sim 80 \%$ humidity or a living modified organism-controlled paddy field (Kyung Hee University, Suwon, South Korea) for the phenotype analysis. Anthers from the WT (DJ) plants were harvested at different developmental stages for quantitative realtime polymerase chain reaction (qRT-PCR) analysis as previously described (Moon et al., 2019).

\section{Multiple Sequence Alignment and Phylogenetic Tree Construction}

To perform phylogenetic analysis of the ANTH proteins in rice and Arabidopsis, we collected protein sequences with locus ID from the Rice Genome Annotation Project ${ }^{1}$, National Center for Biotechnology Information $\left(\mathrm{NCBI}^{2}\right)$, and Phytozome platform ${ }^{3}$. Multiple alignment of the amino acid sequences was performed using ClustalW. The phylogenetic analysis was performed using MEGA 7.0.26 with maximum likelihood and neighbor-joining methods (bootstrap repeat was 1,000 ) as previously described (Kim et al., 2019).

\section{Meta-Expression Analysis}

We used a publicly available rice Affymetrix microarray data set prepared from anthers and pollen from the NCBI Gene Expression Omnibus (GEO) (Hong et al., 2019) to identify

\footnotetext{
${ }^{1}$ http://rice.plantbiology.msu.edu/

${ }^{2}$ https://www.ncbi.nlm.nih.gov/

${ }^{3}$ https://phytozome.jgi.doe.gov/pz/portal.html
} 
late pollen-preferred genes. To examine expression patterns, we used the Affy package encoded by $\mathrm{R}$ language to normalize the signal intensity and then transformed them into $\log 2$ values. The normalized data, including averaged Affymetrix anatomical meta-expression data, were then used for further investigation, i.e., heatmap construction and identification of the late pollenpreferred genes (Nguyen et al., 2015).

Microarray data, including Affymetric and Agilent array data, were downloaded from the NCBI $\mathrm{GEO}^{4}$ and Genevestigator ${ }^{5}$ (Zimmermann et al., 2004; Cao et al., 2012). We then uploaded the normalized data to the Multi Experiment Viewer ${ }^{6}$ and visualized the data using heatmaps. We used Genevestigator to compare the levels of gene expression in several organs and to estimate the functional similarity among rice and Arabidopsis members.

\section{RNA Extraction, cDNA Construction, and qRT-PCR}

Tissue samples, including pollen of rice (Oryza sativa cv. Dongjin) grown in paddy fields, were frozen in liquid nitrogen and homogenized with a TissueLyser II (Qiagen, Hilden, Germany). Total RNA was extracted using RNAiso Plus according to the manufacturer's protocol (TakaraBio, Kyoto, Japan). cDNAs were synthesized using a SuPrimeScript RT premix from GeNet Bio (Chungcheongnam-do, South Korea) (Vo et al., 2015). qRT-PCR was performed with a Qiagen RotorGene Q qRT-PCR cycler (Qiagen) using the following thermal cycling procedure: $95^{\circ} \mathrm{C}$ for $10 \mathrm{~s}, 60^{\circ} \mathrm{C}$ for $30 \mathrm{~s}$, and $72^{\circ} \mathrm{C}$ for $1 \mathrm{~min}$. To evaluate tissue-specific expression patterns by qRT-PCR, we used rice ubiquitin 5 (OsUbi5, LOC_Os01g22490) (Jain et al., 2006) as an endogenous control to normalize the variance in the amount of cDNA. Gene-specific qRTPCR primers were designed for a specific region of each gene (Supplementary Figure 1 and Supplementary Table 2), and the accuracy and efficiency of each primer set were verified through PCR amplification of the gDNA to optimize the PCR conditions and melting curve. The fold change was calculated using the comparative delta-CT (Pfaffl, 2001; Silver et al., 2006). Three biological replicates were analyzed, and each reaction was performed at least three times with technical replicates.

\section{Subcellular Localization Analysis}

The coding sequences (CDSs) of four OsANTH genes were amplified from mature anther cDNA and cloned into pGreen vectors fused with $\mathrm{C}$-terminal green fluorescence protein (GFP). All the cloning primers used in the experiments are listed in Supplementary Table 2. The constructs were transfected into Agrobacterium tumefaciens strain GV3101 and used for Nicotiana benthamiana infiltration. Two to three days after infiltration, GFP fluorescence was observed with a confocal laser scanning microscope (Zeiss LSM 510; Zeiss, Oberkochen, Germany) using 488/505-530 nm excitation/emission filter sets. Fluorescence images were digitized using the Zeiss LSM image browser.

\footnotetext{
${ }^{4}$ http://www.ncbi.nlm.nih.gov/geo/

${ }^{5}$ https://genevestigator.com/gv/

${ }^{6}$ http://mev.tm4.org/
}

To identify the subcellular localization in the cell membrane, the tobacco leaves were stained with FM4-64 (Thermo Fisher Scientific, Waltham, MA, United States). Tobacco leaf discs were immersed in $0.1 \%$ FM4-64 solution for more than 15 min under dark conditions and observed in a red fluorescence protein (RFP) channel using $558 \mathrm{~nm}$ excitation/emission filter sets. Brefeldin A (BFA) treatment was performed for approximately an hour after the tobacco leaf was immersed in $10 \mu \mathrm{M}$ BFA.

\section{Isolation and Analysis of Mutant Plants}

A T-DNA insertional mutant line of OsANTH3 was isolated from the T-DNA insertional mutant population in the PFG library (Hong and Jung, 2018). Genotypes were determined via PCR in $50 \mu \mathrm{L}$ of a mixture containing $20 \mathrm{ng}$ of genomic DNA, 10X e-Taq buffer, $0.2 \mathrm{mM}$ dNTP, $0.5 \mathrm{U}$ of e-Taq polymerase (Solgent, Daejeon, Korea), and $1 \mathrm{mM}$ of the primers. The protocol included 35 cycles at $94^{\circ} \mathrm{C}$ for $60 \mathrm{~s}, 60^{\circ} \mathrm{C}$ for $60 \mathrm{~s}$, and $72^{\circ} \mathrm{C}$ for $120 \mathrm{~s}$. The primers used for genotyping were $5^{\prime}$-CACATCTGGGTGGGAGCTTG$3^{\prime}$ (3,276 bp downstream from the ATG start codon of the ANTH3 gene), 5'-GCCATTGGTACGAGGTCACC-3' (4,101 bp downstream from the ATG start codon of ANTH3 gene), and $5^{\prime}$ AACGCCTGATCAATTCCACAG-3' (80 bp downstream from the ATG start codon of the GUS gene).

Additional mutant alleles were generated using the CRISPR/Cas9 system. The specific target sequences were identified using the online CRISPRdirect program (Naito et al., 2015). The oligomers were cloned into the BsaI site of the pRGEB32 vector, which was then used to transform the WT rice via Agrobacterium (Kim E. et al., 2020). At least two regions were selected for gene editing, and approximately 20 hygromycinselected lines were analyzed. Gene editing was confirmed by Sanger sequencing.

Histochemical GUS-staining was performed as described by Moon et al. (2019). The assayed flowers and pollen grains were photographed with an Olympus BX61 microscope (Olympus, Tokyo, Japan).

\section{Cytological Analysis of Pollen Germination}

Flowers and anthers were photographed with a SZX61 microscope (Olympus). To investigate pollen maturation, spikelets were collected before anthesis, and the pollen grains were squeezed out with tweezers and stained with $1 \% \mathrm{I}_{2}$-KI.

To observe the in vitro pollen germination percentage and morphology of pollen tubes, fresh pollen grains collected just after anthesis were placed directly onto a solid or liquid pollen germination medium (PGM). The PGM was freshly prepared with $20 \%(\mathrm{w} / \mathrm{v})$ sucrose, $10 \%$ polyethylene glycol $4000,3 \mathrm{mM}$ calcium nitrate, $40 \mathrm{mg} / \mathrm{L}$ boric acid, and $10 \mathrm{mg} / \mathrm{L}$ vitamin $\mathrm{B} 1$ ( $\mathrm{pH}$ 6.8-7.0) (Kim E. et al., 2020). The solidified PGM slide with $1 \%$ agarose was covered with a cover glass and incubated in a moist chamber at $28^{\circ} \mathrm{C}$ in the dark for $20 \mathrm{~min}$. The germination percentage was analyzed in triplicate with at least 150 pollen grains in each examination. 
Ikarugamycin (IKA) has been used as a CME inhibitor in plants and animals, although its mode of action remains to be determined (Elkin et al., 2016). For the IKA treatment, different concentrations $(10,50$, and $100 \mu \mathrm{M})$ were prepared in PGM using a $10 \mathrm{mM}$ IKA stock solution prepared in dimethyl sulfoxide (DMSO). In addition, pollen grains were germinated in the presence of DMSO as control. Working concentrations of DMSO were $<0.2 \%(\mathrm{v} / \mathrm{v})$.

\section{Yeast Two Hybrid Analysis}

The full length, N-terminal $(1 \sim 1,095 \mathrm{bp})$, and C-terminal (1,096 end) CDSs of ANTH3 were cloned at the BamHI site of the pGADT7-Rec vector. The full-length, N-terminal, and C-terminal CDSs of GORI were cloned at the EcoRI/BamHI site of the pGBKT7 vector (Kim et al., 2021). The C-terminal (633end) CDS of OsPME31 (Kim Y. et al., 2020) was cloned into the pGBKT7 vector. To test the self-transcriptional activity of the GORI bait, the plasmid was introduced with an empty prey vector into the yeast strain AH109. Yeast transformants of the bait and prey plasmids were selected on a synthetic defined (SD) minimal medium lacking leucine and tryptophan (SD-LW) and were replica-plated onto various SD selection media, including media lacking leucine, tryptophan, and histidine (SD-LWH, +5 $\mathrm{mM}$ 3-AT) and media lacking leucine, tryptophan, and adenine (SD-LWA).

\section{RESULTS}

\section{Identification of 17 OsANTH Genes in Rice}

To obtain all ANTH domain-containing sequences in rice, we reannotated protein sequences from the Rice Genome Annotation Project ${ }^{1}$ on the Pfam database (PF07651.9). A total of 17 genes were identified as putative $A N T H$ genes in rice and named OsANTH1 to OsANTH17 based on their physical location on the chromosomes (Supplementary Table 1). To further understand the structure of the OsANTH genes, we compared the corresponding genomic DNA sequences and obtained the exon and intron structures of the OsANTH genes (Figure 1A). Intron numbers varied from 0 to 15 .

All candidate OsANTH proteins were analyzed using SMART and Pfam tools, indicating that they all possessed the ANTH domain at the $\mathrm{N}$-terminal region of the protein and exhibited various protein sizes (Figure 1B). OsANTH9 was the smallest protein with 116 amino acids, and OsANTH15 was the largest protein with 863 amino acids.

\section{Phylogenetic Tree and Motif Composition Analyses of OsANTH Proteins}

Metazoan and fungal genomes have fewer ANTH proteins than plants, implying that the ANTH proteins in plants have evolved more divergent functions than those in non-plant systems (Zouhar and Sauer, 2014). To understand the evolutionary relationships of ANTH proteins in plant species, the amino acid sequences of 17 ANTH proteins in rice and 18 ANTH proteins in Arabidopsis (PICALMs) were isolated and aligned (Figure 2A). The ANTH families of rice and Arabidopsis are composed of a similar number of family members, which seems to be due to similar evolutional progress. Arabidopsis and rice members are not clustered according to species, and instead, many members seem to have coevolved into monocots and dicots and duplicated. However, some members seem to be restricted to either Arabidopsis or rice. For example, OsANTH13 and OsANTH15 only exist in rice, while three PICALM10 homologs do not exist in rice (Figure $\mathbf{2 A}$ ).

Mammalian ANTH proteins are divided into subfamily groups depending on the presence of actin-binding motifs. However, plant ANTH proteins do not have an I/LWEQ sequence, which is an actin-binding motif. Instead, they are divided into two subgroups based on the presence of asparagineproline-phenylalanine (NPF) motifs: the NPF-rich and NPF-less subfamilies (Holstein and Oliviusson, 2005; Zouhar and Sauer, 2014). NPF motifs bind to Eps15 homology (EH) domaincontaining proteins that function as regulators of endocytosis through their ability to interact with other proteins involved in CME. Our analysis demonstrated that the NPF-rich subfamily had 14-15 exons with a corresponding number of introns, while the NPF-less subfamily had few or no introns in rice (Figure 1A). In addition, the exon-intron structures of the closely linked genes in the phylogenetic tree were almost identical.

Plant ANTH proteins contain variations of the conserved mammalian ANTH signature motif (Ford et al., 2001), namely $\mathrm{KAT}(\mathrm{X}) 5 / 6 \mathrm{P}(\mathrm{X}) 3 \mathrm{~K} / \mathrm{RH} / \mathrm{Y}$, which enables ANTH proteins to bind to the PM. The ANTH families of both rice and Arabidopsis have ANTH domains in the N-terminal region (Figure 2B). However, OsANTH9, OsANTH13, OsANTH15, and OsANTH16 contained shorter ANTH domains than the other family members (Figure 1B), and the ANTH consensus region was not well conserved with other OsANTH protein sequences. Using the rice male gamete expression database $\left(\mathrm{RMEDB}^{7}\right)$ to check the expression, these putative OsANTH genes were rarely expressed and may be pseudogenes that lost their function during evolution (Supplementary Figure 3). Protein alignment analysis of 13 OsANTH genes, excluding the abovementioned 4 OsANTH genes, showed that the ANTH signature motif is well conserved between the $\alpha$-helix 1 and $\alpha$-helix 2 motifs, except for partial conservation in OsANTH11 and OsANTH14 (Supplementary Figure 2).

Relatively, the C-terminal sequences of most ANTH domaincontaining proteins are diversified (Supplementary Figure 2) and are known to interact with other components of the vesicle-generating machinery, such as clathrin (LegendreGuillemin et al., 2004; de Craene et al., 2012). In this respect, ANTH domain-containing proteins act as a bridge between $\mathrm{PIP}_{2}$ and several components of the CME machinery. Endocytosis is a complex molecular process that depends on regulated interactions between a variety of proteins and lipids through specific modules. Excluding OsANTH9, OsANTH13, and OsANTH17, the C-terminal of the remaining $14 \mathrm{ANTH}$

\footnotetext{
${ }^{7}$ http://ricephylogenomics-khu.org/RMEDB/home.php
} 
A

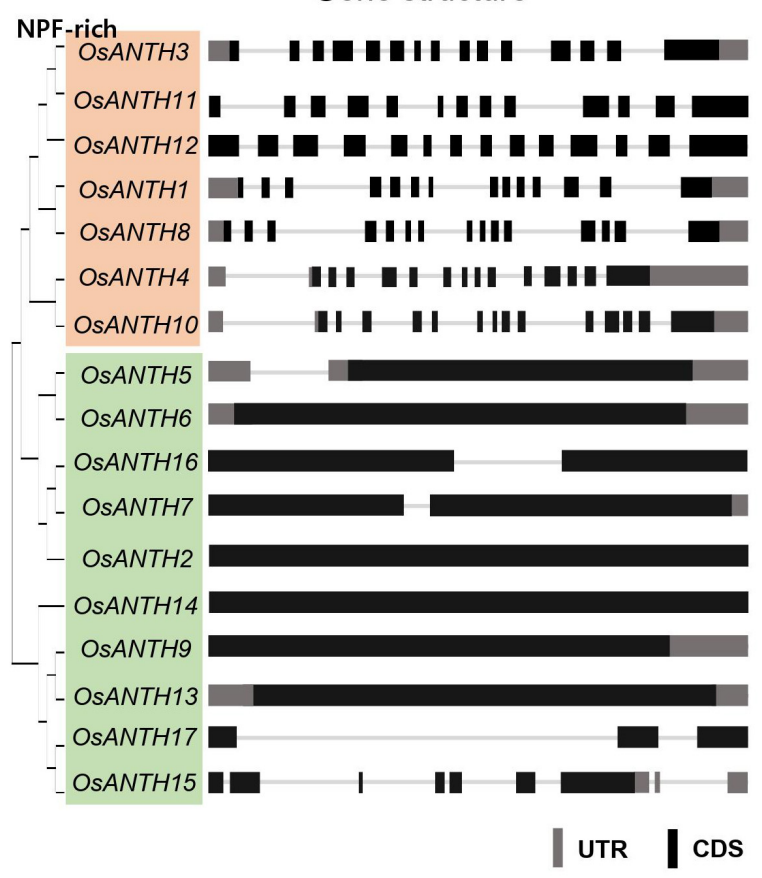

B
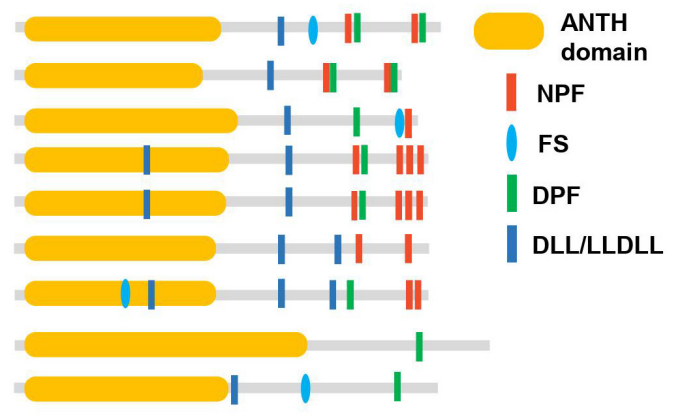

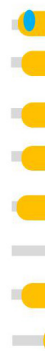

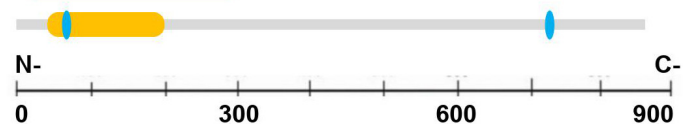

FIGURE 1 | Gene structure and motif pattern of rice AP180 N-terminal homology (ANTH) proteins. (A) Phylogenetic tree and gene structure of seventeen ANTH genes. Upper 7 and lower 10 ANTH proteins are divided into two subgroups according to gene structure (whether they contain no or only a few introns or many introns). Black and gray boxes indicate coding sequences (CDSs) and untranslated regions (UTR), respectively, and lines represent introns. (B) Motif analysis of ANTH proteins: ANTH, asparagine-proline-phenylalanine (NPF), the clathrin-binding motif aspartic acid-leucine-leucine/leucine-leucine-aspartic acid-leucine-leucine (DLL/LLDLL), the clathrin-binding motif phenylalanine-Serine (FS), and the $\alpha$-adaptin-binding motif aspartic acid-proline-phenylalanine (DPF). Bar in each section represents 100 aa.

proteins possessed binding sites for various proteins including $\mathrm{EH}$, clathrin, and the EAR-binding domain. The presence of the mammalian clathrin-binding motifs DDL and FS, as well as the $\alpha$-adaptin-binding motif DPF (Brett et al., 2002), within the OsANTH protein sequences (Figure 1B) indicated conserved motifs of ANTH in plants and animals. In contrast to their yeast and mammalian counterparts, OsANTH proteins do not contain conserved ubiquitin-interacting motifs, such as the DPW and FXDXF motifs or the I/LWEQ motif, which are required for interaction with ubiquitylated proteins using the $\alpha$-adaptin of AP- 2 or the actin cytoskeleton, respectively (Holstein and Oliviusson, 2005).

\section{Expression Pattern Analysis of OsANTH Genes}

To identify possible ANTH candidates related to pollen tube growth in rice, we used the RMEDB database, which provides meta-expression analysis focused on rice male gamete development (Chandran et al., 2020). The meta-expression data in the context of the phylogenetic tree were produced to check the function of OsANTH genes with respect to anther and pollen development compared with other tissues/organs (Figure 3A). In addition, we examined their expression profiles in RNAsequencing samples (Supplementary Figure 3; Hong et al., 2020).
We found that the expression patterns of five genes (OsANTH3, OsANTH11, OsANTH7, OsANTH2, and OsANTH14) were closely associated with late pollen development, i.e., at the tricellular pollen grains, mature pollen, and germinated pollen stages. OsANTH3, OsANTH11, OsANTH2, and OsANTH7 had similar expression patterns. Especially, OsANTH3 and OsANTH11 had high expression in the late pollen development stage and were located in the same sister node of the phylogenetic tree. Conversely, OsANTH8, which was in the same sister node as OsANTH3 and OsANTH11, showed high expression in not only late pollen development but also in somatic tissues as well. Therefore, OsANTH8 is expected to have a role in various tissues including pollen and somatic tissues, while five OsANTH genes are expected to be specific to pollen function.

Further, to verify the meta-expression data, we performed qRT-PCR using eight tissues: shoot, root, seed, anthers containing meiosis microspore, tetrad microspore, young microspore, vacuolated pollen, and mature pollen (Figure 3B). Similar to the microarray results, OsANTH3, OsANTH11, OsANTH7, OsANTH2, and OsANTH14 showed preferential expression in the mature pollen-containing anther but were not significantly expressed in other tissues. Therefore, these five genes (OsANTH3, OsANTH11, OsANTH7, OsANTH2, and OsANTH14) may play key roles in pollen tube growth and other processes that occur after pollen maturation. OsANTH3 and OsANTH11 

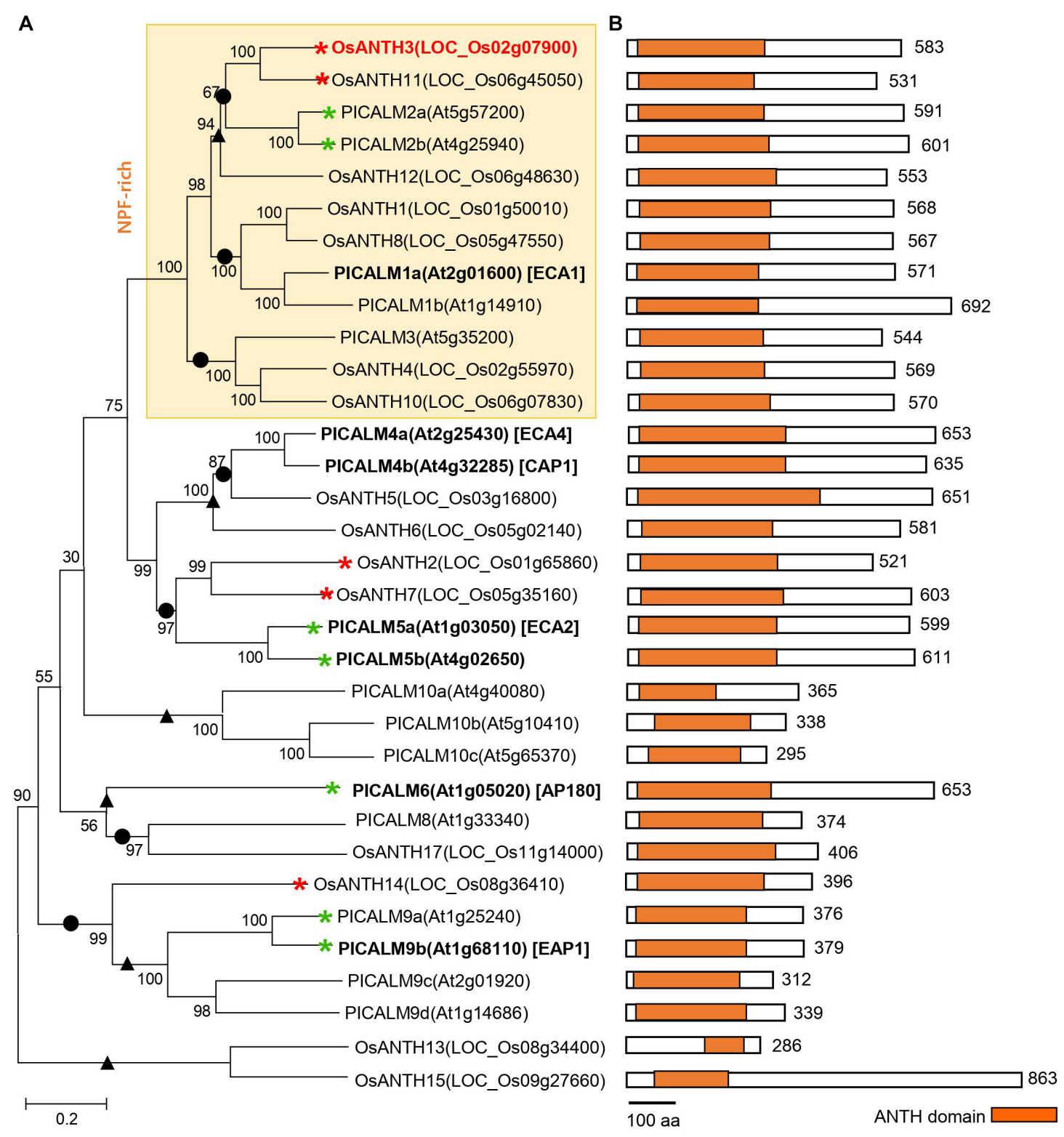

FIGURE 2 | Phylogenetic analysis of the AP180 N-terminal homology (ANTH) family from Oryza sativa and Arabidopsis thaliana. (A) Phylogenetic tree constructed using the protein sequences for each gene with MEGA7 software. Red and green asterisks indicate ANTH genes with pollen-preferential expression in rice (in this study) and Arabidopsis (Muro et al., 2018; Supplementary Figure 6), respectively. Bold letters indicate the functionally characterized genes, and red color indicates genes for which the mutant phenotype was analyzed in this study. We used 1,000 bootstrap replicates, and the number at each node is a bootstrap value. $\bullet$ and $\mathbf{\Delta}$ indicate branches conserved and diversified between Arabidopsis and rice, respectively. (B) Schematic diagram of the ANTH protein primary structure with the ANTH domain. The length of each ANTH protein is shown on the right regarding the number of amino acids (aa). The scale bar indicates 100 aa. Orange boxes indicate the position and length of the conserved ANTH domain in the family. Each domain is presented in the N-terminal of the protein. OsANTH9 and OsANTH16 are not aligned because the gene length is too short.

belong to the NPF-rich group, while OsANTH7, OsANTH2, and OsANTH14 belong to the NPF-less group (Figure 1B). OsANTH3 and OsANTH11, which belong to the NPF-rich group, have a similar expression pattern. These two genes were duplicated since the divergence of Arabidopsis and may be functionally redundant. Especially, OsANTH3 showed the highest level of expression among the five genes in the mature pollen.
To investigate the in planta expression of the OsANTH3 gene in rice, the expression of GUS under the control of the OsANTH3 promoter was investigated (Figure 3C). GUS expression appeared in the pollen of the anthers. In particular, OsANTH3 showed peak expression in the late pollen stages, including in mature pollen and germinating pollen, after initial expression in the bicellular pollen stage (Figure 3C). In germinated pollen, GUS was expressed in the pollen tube as well 
A

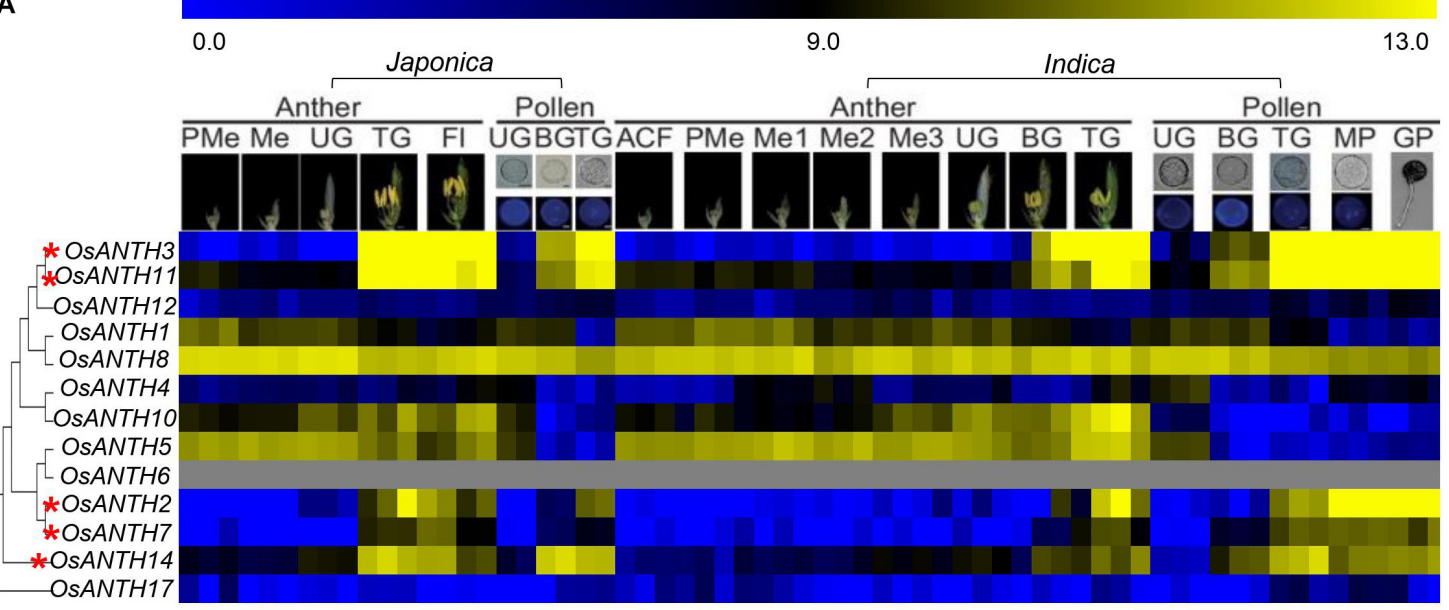

B

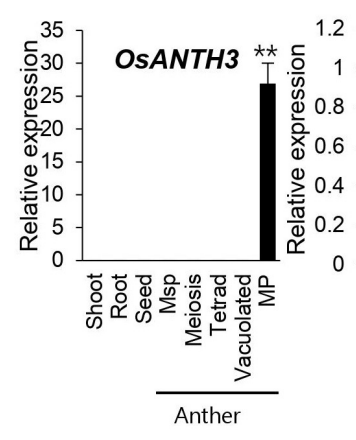

C

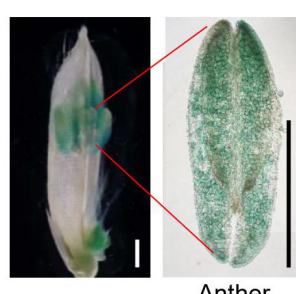

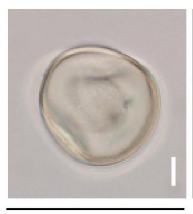

UG
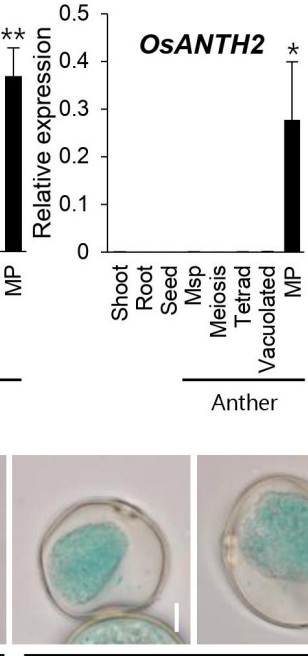

.

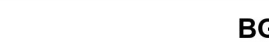

BG
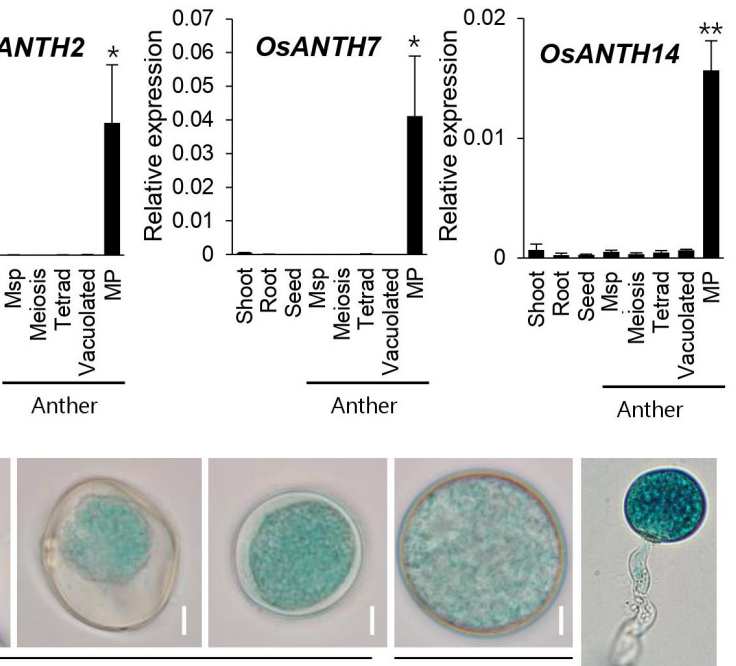

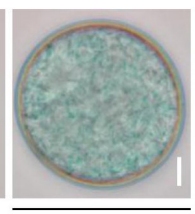

MP

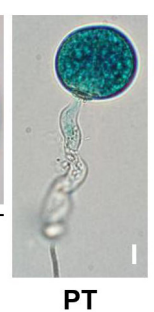

FIGURE 3 | Expression patterns of OSANTH genes in rice. (A) Heatmap showing expression levels as determined by microarray analysis. Yellow color in the heatmap indicates a high level of expression, whereas dark blue indicates low expression. Numeric values indicate an average of the normalized log 2 intensity value of the microarray data. Pollen-preferentially expressed OSANTH genes are indicated by red asterisks. ACF, archesporial cell-forming stage; BG, bi-cellular gametophyte stage; Fl, flowering stage; GP, germinated pollen; Me, meiotic stage; Me1, meiotic leptotene stage; Me2, meiotic zygotene-pachytene stage; Me3, meiotic diplotene-tetrad stage; MP, mature pollen stage; PMe, pre-meiosis; TG, tricellular pollen stage; UG, uni-cellular gametophyte stage. (B) Expression of pollen-preferred ANTH domain-containing protein-coding genes in Oryza sativa (OsANTH genes) analyzed via qPCR in various tissues of rice. Msp, Microspore; MP, mature pollen. Rice ubiquitin 5 (OsUbi5, LOC_Os01g22490) was used as an internal control. The $y$-axis shows the expression level relative to OsUbi5, while the $x$-axis shows the samples used for analyses. Error bars represent the standard errors of three biological replicates. Significant differences are indicated by asterisks; ${ }^{*} p<0.01$ and ${ }^{* *} p<0.0001$. Data were analyzed by employing one-way analysis of variance with repeated measures using Tukey's pairwise comparison test. (C) Expression pattern of OSANTH3-GUS in transgenic rice plants at the anther and sequential stages of pollen development. UG, unicellular gametophyte stage; BG, bicellular gametophyte stage; MP, mature pollen stage. Bar in flower and anther image = $1 \mathrm{~mm}$; bar in pollen image = $10 \mu \mathrm{m}$.

as the pollen grain. It can be expected that OsANTH3 may play a role in pollen germination or pollen tube elongation.

\section{Subcellular Localization of Four ANTH Proteins}

ANTH proteins are known to assemble clathrin to the PM to initiate CME (Kaneda et al., 2019). In Arabidopsis, PICALM5a,
PICALM5b, and PICALM6 have been shown to localize apical PM and the cytosol of pollen tubes (Barth and Holstein, 2004; Muro et al., 2018). To evaluate whether the OsANTH proteins are also localized at the PM, we constructed GFP-fused OsANTH proteins driven by the CaMV $35 \mathrm{~S}$ promoter and examined their subcellular localization in Agrobacterium-infiltrated tobacco epidermal cells. The experiment was conducted with four pollen-preferred genes (OsANTH3, OsANTH11, OsANTH2, and 


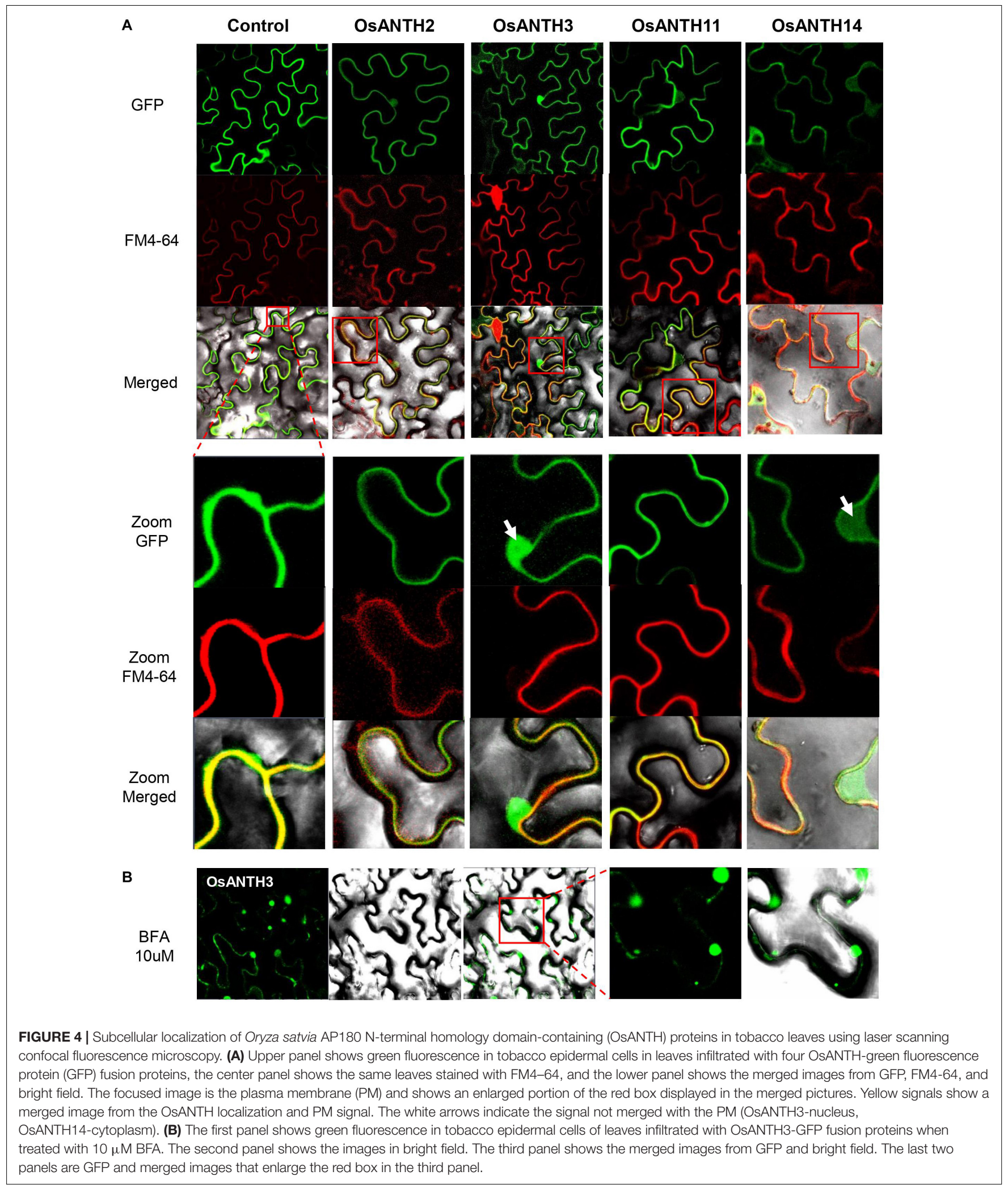

OsANTH14), and all four genes were found to have GFP signals in the nucleus, PM, and cytosol (Figure 4A). When tobacco cells were stained with FM4-64, which is a marker for membrane trafficking in plant cells (Bolte et al., 2004; Wang et al., 2005), the signal from all four OsANTH-GFP proteins overlapped with the signal from the FM4-64 stain. These results 
suggest that four OsANTH proteins with pollen preferential expression are localized in the PM and internalized through endocytosis (Nagawa et al., 2012) when transiently expressed in tobacco leaves.

BFA is a fungal metabolite that inhibits exocytosis but allows the first step of endocytosis to proceed in eukaryotic cells (Lippincott-Schwartz et al., 1991; Baluška et al., 2002; Nebenführ et al., 2002; Geldner et al., 2003). Thus, BFA inhibits the endocytic recycling of $\mathrm{PM}$ proteins. When $\mathrm{BFA}$ is used to treat plant cells, PM proteins or peripheral membrane proteins that are rapidly recycled, are internalized via endocytosis and gather in BFA-induced compartments to form an endosome. Due to these characteristics, BFA has been an important tool for endocytosis research in Arabidopsis (Samaj et al., 2004). We treated the tobacco leaves expressing OsANTH3-GFP with $10 \mu \mathrm{M}$ BFA to determine whether OsANTH was involved in endocytosis. One hour later, we were able to observe several endosomal vesicles in the cytoplasm close to the PM (Figure 4B), while the BFA bodies were not observed with OsANTH11-GFP expression.

\section{Genetic Study Using the Mutation of OsANTH3}

To investigate the possible role of OsANTH protein in pollen, we decided to focus on OsANTH3 as it was preferentially expressed, and expressed at a high level, in pollen. We identified a T-DNA mutant in OsANTH3 in the PFG library and confirmed the line contained a single insert in the 15th exon of OsANTH3 by genotyping (Figure 5A). After isolating the homozygous mutant (Figure 5B), we observed about an $80 \%$ reduced fertility rate in the T-DNA insertional homozygous mutant without other obvious growth defects (Figure 5J). To confirm the defect of OsANTH3 in osanth3-1, we performed qRT-PCR analysis on mature anthers of osanth3-1 and the WT (Figure 5C). Compared with the high expression in WT, we found that OsANTH3 expression was significantly reduced, confirming that the T-DNA insertional mutant has a defect in the function of OsANTH3. We then analyzed the expression of other pollen-preferred OsANTH genes in the mutant. OsANTH2 and OsANTH7 expression slightly but not significantly decreased in the mutant, while expression of OsANTH11 slightly increased, suggesting that OsANTH2 and OsANTH7 were not affected by OsANTH3; however, OsANTH11 could partially compensate for the defect in the mutant.

To find the cause of the partial sterile phenotype of the osanth3-1 homozygous mutant (Figures 5D,E), we further examined phenotypical changes. Mutant plants produce normal panicle (Figure 5F), floral organs, and pollen grains (Figure 5G). However, we found that the germination percentage of pollen in osanth3-1 was $29.38 \%$, compared with $63.67 \%$ in the WT (Figures $\mathbf{5 H}, \mathbf{K})$. The KI-staining results showed no difference between the WT and mutant pollens for maturation (Figure 5G), indicating that that the reduction in the percentage of germination was not caused by a lack of starch in the pollen grain. The size variation of pollen grains indicates the hydration status of rice pollen grains, indicating some smaller grain are not hydrated fully. Although the germination percentage in osanth3-1 pollen was more reduced than that in WT pollen, germinated osanth3-1 pollen normally grows pollen tubes (Figures 5H,I). The homozygous mutant generated by CRISPRCas9, named osanth3-2, also showed a similar phenotype (Supplementary Figure 5), confirming the role of OsANTH3 in pollen germination. Our study reveals that OsANTH3 plays a role in rice pollen germination.

To further examine how CME affects germination, we treated a CME inhibitor, IKA. As the concentration of IKA increased from 0 to $100 \mu \mathrm{M}$ (Figure 6A), the germination percentage decreased, suggesting that $\mathrm{CME}$ can affect rice pollen germination. Compared with the WT, osanth3-1, which does not normally perform CME due to a lack of ANTH3 function, showed a low germination percentage regardless of IKA concentration (Figure 6B). The results suggest that CME affects rice pollen germination and is mediated by OsANTH3.

\section{DISCUSSION}

ANTH proteins act as adaptor proteins for CME to recycle or degrade cargo proteins in the membrane. In this study, we explored the physiological role of CME and ANTH genes regarding pollen germination in rice. We found 17 OsANTH genes in the rice genome; four genes contained a partial ANTH domain and were expressed at undetectable levels.

ANTH proteins contained a conserved $\mathrm{PIP}_{2}$-binding site in the $\alpha$-helix 2 and $\alpha$-helix 3 motifs at the $\mathrm{N}$-terminal region (Supplementary Figure 2), due to which the proteins bind to the PM (Holstein and Oliviusson, 2005). Their secondary structure in the N-terminal region was also very similar (Supplementary Figure 6). The C-terminal region of OsANTH proteins, which contains various of motifs, was predicted to be exposed to the cytoplasm, as a general feature of the ANTH protein family. Therefore, this region may be able to induce the recruitment of coat component and clathrin assembly. When expressed in tobacco leaves, signals from the four GFP-OsANTH fusion proteins (OsANTH2, OsANTH3, OsANTH11, and OsANTH14) with pollen preferential expression were observed in the PM, cytosol, and nucleus (Figure 4). Similar to our results, the ANTH proteins in Arabidopsis (PICALM5a and PICALM5b) are localized in mature pollen grains and the cytoplasm and PM of germinated pollen tubes (Muro et al., 2018). Localization of ANTH proteins in the nucleus has not yet been reported in plants; however, in animals, the epsin protein has been reported to be translocated from the cytoplasm to the nucleus, suggesting that it may convey signals from the endocytic pathway to the transcription machinery (Hurtley, 2000).

Members of the ANTH family are also found in most taxa, excluding the Chromista and Excavata. The Plantae ANTH family has evolved to be more subdivided than other kingdoms (Opisthokonta, Amoebozozoa, and Euglenozoa). Plantae, which undergo endocytosis and endosomal sorting, all had a complex evolution, as illustrated by the high number of duplications in each subfamily. In most plants, the molecular mechanisms and how this differs in different tissues are not well characterized. However, this increased number of ANTH genes suggests that 


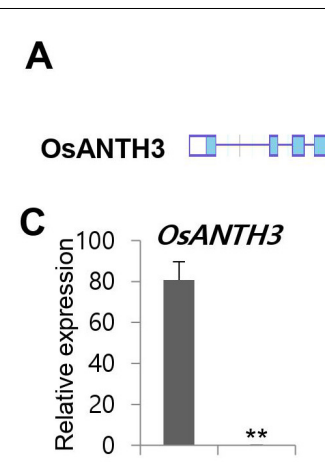

DJ osanth3-1
3A-10743

osanth3-1 $\mathrm{BR}$ GUS $\rightarrow \mathrm{BL}$

$\mathrm{N}$

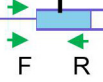

B

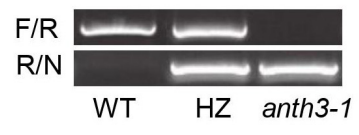

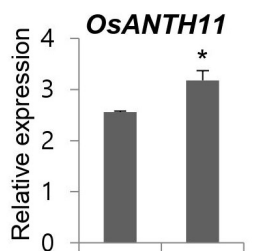

DJ osanth3-1

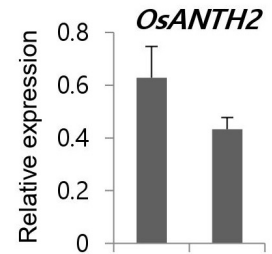

DJ osanth3-1

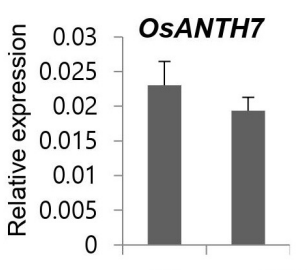

DJ osanth3-1
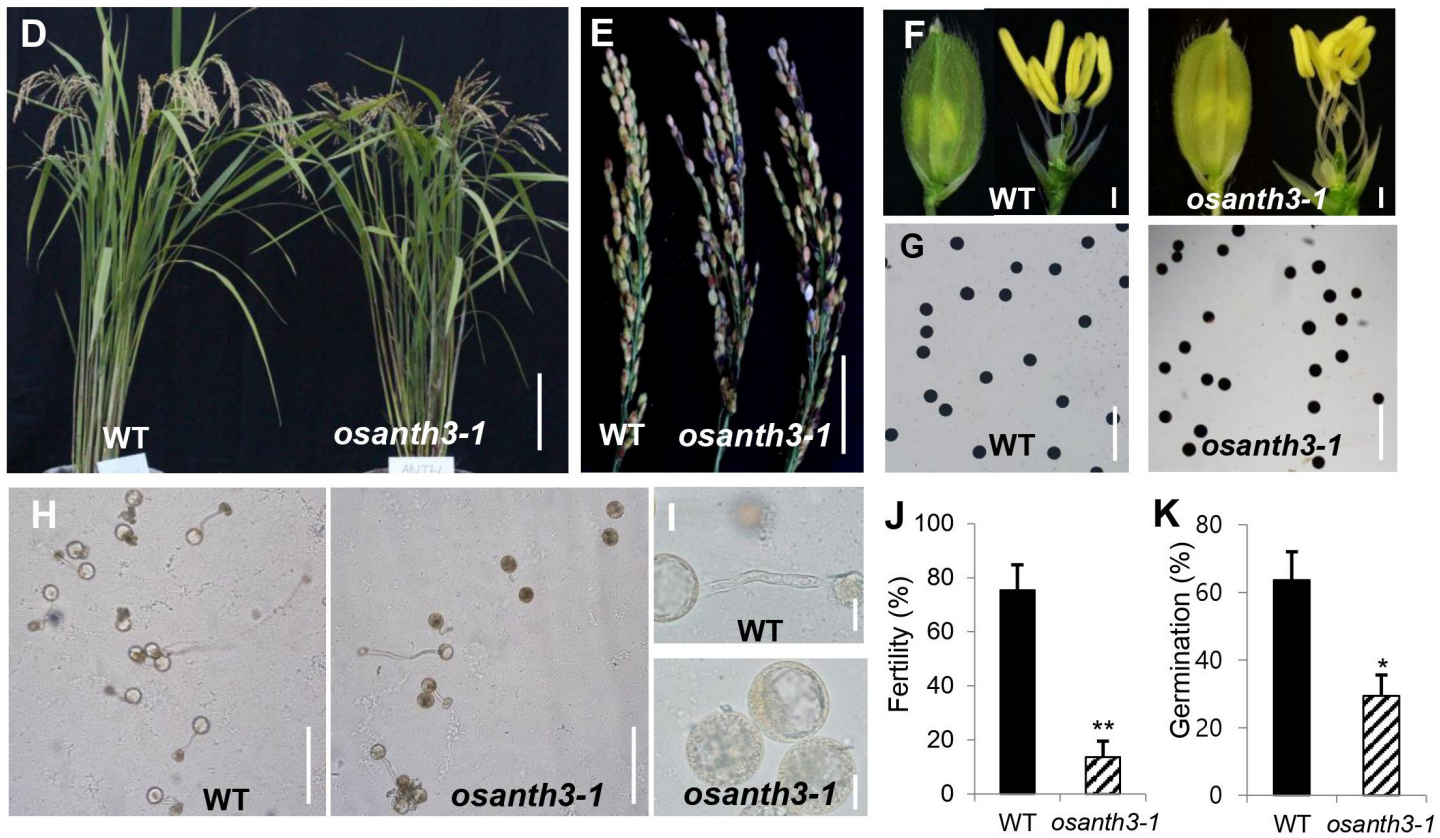

FIGURE 5 | Phenotypes of plants containing mutations in AP180 N-terminal homology (ANTH) domain-containing protein-coding gene 3 in Oryza sativa (OsANTH3) (LOC_Os02g07900). (A) Schematic diagram of the osanth3-1 mutant with T-DNA insertion. (B) Polymerase chain reaction (PCR) analysis for genotyping using two primer sets (F/R and R/N). Genotyping results showed that osanth3-1 was homozygous. (C) qRT-PCR for four genes (OsANTH3, OsANTH11, OsANTH2, and OsANTH7) in Dongjin (WT) and osanth3-1. Rice ubiquitin 5 (OsUbi5, LOC_Os01g22490) was used as an internal control. The $y$-axis shows the expression level relative to OsUbi5, while the $x$-axis shows the samples used for analyses. Error bars represent the standard errors of three biological replicates. (D) Vegetative growth was not significantly different between the WT and osanth3-1, but osanth3-1 shows partial male sterility. (E) Panicle of the osanth3-1 mutant observed with many empty grains due to poor germination. (F) Whole spikelet (left side) and spikelet after removing lemma and palea (right side) of osanth3-1 are similar to those of the WT. (G) Pollen grains of the WT and osanth3-1 mutant stained with KI solution exhibited normal starch accumulation. (H,I) Microscopic photograph of pollen germination in the WT and osanth3-1 mutant. Compared with that in the WT, osanth3-1 exhibited a lower germination percentage (J). Fertility rate was calculated as (average of the number of seeds normally germinated)/(total seeds) $\times 100$ for each panicle. (K) The germination rate was calculated as (the average of the number of germinated pollen)/(total pollen) $\times 100, n=100$. Significant differences are indicated by asterisks; ${ }^{*} p<0.01$ and ${ }^{* *} p<0.0001$. Scale bars, $10 \mathrm{~cm}$ in (D); $5 \mathrm{~cm}$ in (E); $10 \mu \mathrm{m}$ in $\mathbf{( F )}$; and $20 \mu \mathrm{m}$ in $\mathbf{( I )}$; Bars = $200 \mu \mathrm{m}$ in $\mathbf{( G , H ) . ~ E r r o r ~ b a r s ~ r e p r e s e n t ~ t h e ~ s t a n d a r d ~ e r r o r s ~ o f ~ t h r e e ~ b i o l o g i c a l ~ r e p l i c a t e s ~}(\mathbf{C}, \mathbf{J})$.

endocytosis and endosomal functionality might be specialized to different cell types or cargoes, and these genes have different function at different steps of trafficking in Plantae (de Craene et al., 2012). Even with close evolutionary relationships, each gene might have unique roles in each species (Zouhar and Sauer, 2014) or can be responsible for specified endocytic functions in different tissues. It has been reported that nine ANTH member homologs exist in monocots and dicots, two members are specific to dicots, and seven members are specific to the Brassica family (Zouhar and Sauer, 2014). It is noteworthy that some members seem to have coevolved in monocots and dicots for pollen function. For example, pollen-expressed OsANTH3 and OsANTH11 are homologous to PICALM2a and PICALM2b in Arabidopsis, which are highly expressed in pollen (Supplementary Figure 4). Until now, there have been few reports on the function of ANTH genes in other species besides Arabidopsis. Therefore, further studies are required to determine whether pollen-preferred $A N T H$ genes function as different adaptors during pollen germination.

ANTH proteins can be divided into two subgroups, and the C-terminal region of the NPF-rich subgroup was more conserved 

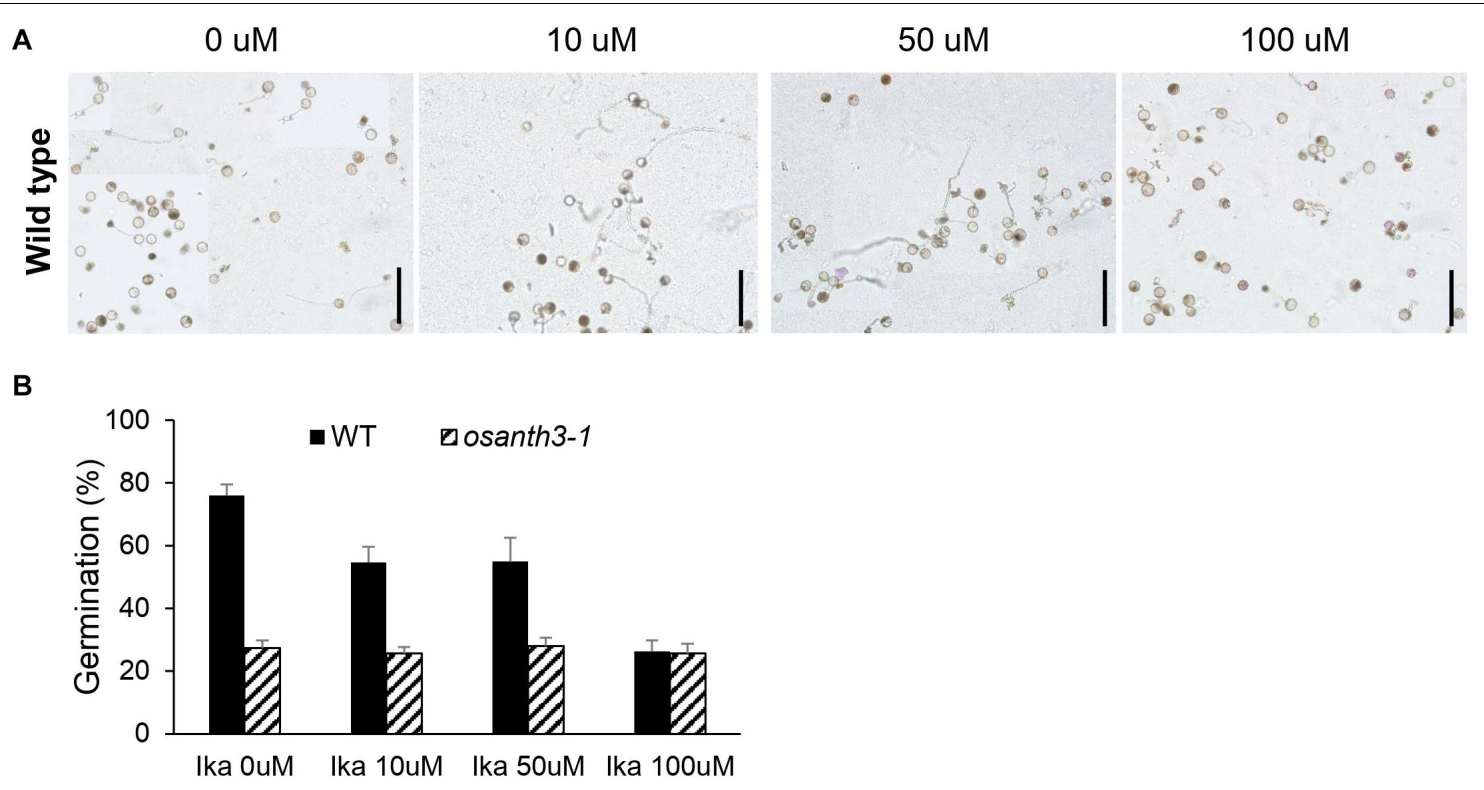

FIGURE 6 | Rice pollen germination percentage and clathrin-mediated endocytosis patterns under Ikarugamycin (IKA) treatment at varying concentrations. (A) Images of rice pollen placed in germination media with different concentrations of IKA (0, 10, 50, and $100 \mu \mathrm{M})$. All media contained $0.2 \%$ dimethyl sulfoxide (DMSO). Bars = $200 \mu \mathrm{m}$. (B) Germination percentage of the wild-type and osanth3-1 plants at different IKA concentrations. Germination rate was calculated with three replicates (each of the replicates was calculated for $n>100$ ).

than that of the NPF-less subgroup (Figure 1). OsANTH3 and OsANTH11 genes belonging to the NPF-rich subgroup were pollen-preferred genes (Figure 1). Few studies have been conducted on the NPF-rich groups in Arabidopsis, and all the genes studied in relation to pollen germination and tube growth belonged to NPF-less groups.

Like the ANTH proteins in other plant species, OsANTH proteins lack the DPW and FXDXF motifs, which are present in animals ANTH protein for $\alpha$-adaptin binding (Owen et al., 1999; Brett et al., 2002). Instead, the NPF and DPF motifs can recruit $\mathrm{EH}$ domain-containing proteins, bind $\alpha$-adaptin, and function as clathrin-binding motifs (Scheele et al., 2001). This suggests that NPF-rich OsANTH3 not only combine with $\alpha$-adaptin to participate in CCV but also function as a clathrinbinding motif. The main endocytic clathrin adapter AP-2 alone is not sufficient to generate CCVs (Brod et al., 2020). CALM and AP180, the animals ANTH proteins, are abundant in CCVs similar to AP-2 (Prasad and Lippoldt, 1988; Skruzny et al., 2015). Additionally, it has been proposed that ANTH proteins act as specific monomeric cargo adapters that recruit cargo to the CCV nucleation site independent of heterotetrameric AP-2 (Holstein and Oliviusson, 2005). For example, AP180 regulates synaptic vesicle size and was shown to regulate the abundance of glucose-dependent insulinotropic polypeptide 1 receptors at postsynaptic elements in Caenorhabditis elegans, and disabled-2 targets low-density lipoprotein receptors for endocytosis (Miller and Lefkowitz, 2001; Burbea et al., 2002; Mishra et al., 2002). Furthermore, huntingtin-interacting protein 1 seems to function as an adaptor for a glutamate receptor in animals (Metzler et al., 2007), and PICALM5 in Arabidopsis proteins serves as a specific loading adaptor to recycle ANXUR proteins (Muro et al., 2018).
In our motif analysis, all seven OsANTH proteins belonging to the NPF-rich group had DLL motifs in the same region, suggesting that they can function in regular endocytic CCVs. Although whether OsANTH is the only protein that assembles clathrin and how it interacts with binding proteins still need to be verified, this suggests that OsANTH is an important factor as a monomeric cargo-specific adaptor for CME (Figure 7).

The TGN in plants is a major sorting system and receives recycled material from endocytosis (Rosquete et al., 2018). The TGN functions as the EE in plant cells (Bolte et al., 2004; Dettmer et al., 2006; Lam et al., 2007). The inhibitor BFA affects membrane traffic in animal secretory and endocytotic pathways (Lippincott-Schwartz et al., 1991). In plants, large intracellular compartments are formed in response to BFA treatment. BFA inhibits the recycling of PM proteins by interfering with secretion from the TGN/EE to the PM in intracellular traffic between them, creating BFA compartments (Lam et al., 2009). In the localization experiment observed on tobacco leaves using the GFP-fusion protein, several endosomal vesicles called BFA bodies were observed around the cytoplasm close to the PM. These results indicate that OsANTH3 prefers to circulate material from the PM to the TGN/EE. In particular, it is assumed that OsANTH3 plays a role in recirculating the signal protein or extracellular material present in the PM, either through endocytosis or by embedding in the PM itself. Integral PM proteins to be internalized via CME are marked for uptake by signal peptides and posttranslational modification like ubiquitination. In plants, it is still unknown how cargo recycles or degrades. Clathrinindependent endocytosis (CIE) appears to be restricted to the tip apex, while CME preferentially occurs in the shoulder region of the apex (Dhonukshe et al., 2007; Moscatelli et al., 2007; 


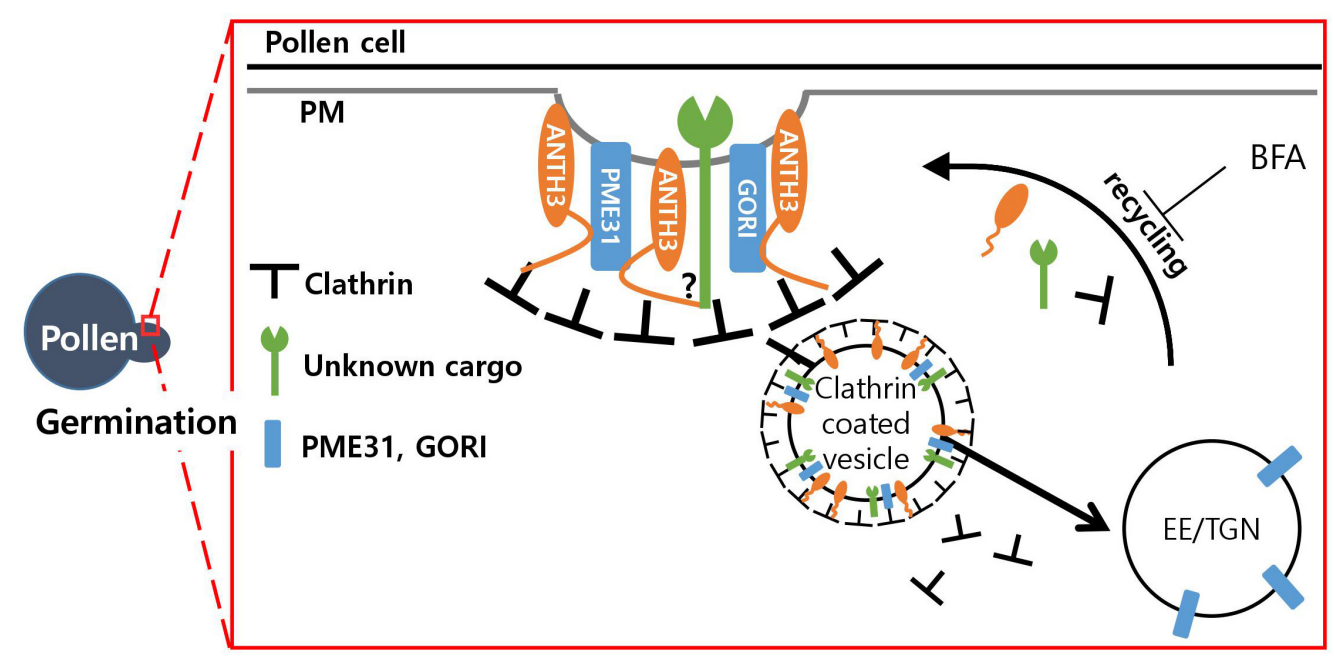

FIGURE 7 | Model of the possible role of Oryza satvia AP180 N-terminal homology domain-containing protein 3 (OsANTH3) in clathrin-mediated endocytosis in germinating pollen. OSANTH3 might function as an adaptor for clathrin-mediated endocytosis, which is critical for rice pollen germination. Unknown cargo and/or GORI and PME31 proteins might be endocytosed from the plasma membrane (PM) to the early endosome/trans-Golgi network (EE/TGN) by interacting with the $\mathrm{N}$-terminal of the OsANTH3 protein. Brefeldin A (BFA) treatment inhibits recycling from the EE/TGN to the PM, and OsANTH3 can be formed as BFA bodies in the cytoplasm.

Moscatelli, 2008; Zhao et al., 2010). IKA, an inhibitor of CME in animal cells, has been used to dissect the endocytic pathway (Hasumi et al., 1992; Luo et al., 2001). In fact, there are reports relating to endocytosis in which the length of pollen tubes was reduced when IKA was used to treat tobacco pollen tubes (Moscatelli et al., 2007). Our results showed that the higher the concentration of IKA on the pollen, the lower the gemination rate, but the pollen tube length of the germinated pollen was found to be normal. This can be explained through the observation of tobacco pollen tubes in response to the IKA treatment; when IKA was processed, endocytosis was not completely inhibited, and the pollen tube gradually reached the normal length. Therefore, even if CME is inhibited, pollen tube growth is possible through CIE. This may be because rice has a relatively short pollen tube length and growth time.

Animal and yeast cells internalize preferential signal molecules through CME in a variety of signal transduction pathways (Goldstein et al., 1985; Wendland et al., 1999). Several studies on Arabidopsis have demonstrated that auxin flow is partially mediated by membrane trafficking-dependent intracellular relocalization and/or degradation of PIN auxin efflux transporters (Kleine-Vehn et al., 2006). In fungus, the CME pathway may be important during germination, germ-tube growth, and initiation of appressorium formation (Atkinson et al., 2002). In plants, most endocytic mechanisms are mediated by the coat protein clathrin. In addition, it has been proven that rapid activation of endocentric processes occurs during the seed germination (Pagnussat et al., 2012). In our study, for the WT under CME-inhibiting conditions, pollen germination was reduced, but not tube elongation. In addition, the results of osanth3-1 showed a reduction in the germination percentage; however, the growth of the pollen tube was normal. This low generation percentage in osanth3-1 eventually led to a decrease in fertility. As a result of drawing the network to find the candidate genes involved in germination signaling using endocytosis and OsANTH3 (Supplementary Figure 7), it was possible to suggest that ANTH could interact with various signaling proteins, including receptor or membrane-integrated proteins involved in endocytosis.

In the $\mathrm{Y} 2 \mathrm{H}$ screening for the identification of direct protein interactions (Supplementary Figure 7), the C-terminal of OsANTH3 (1096 end) was found to interact with the PME31 (pectin methylesterase) (Kim Y. et al., 2020) and GORI proteins (Kim et al., 2021), which are both important for rice pollen germination. GORI encodes a seven WD40 motif protein that is homologous to REN4 in Arabidopsis. Knock-out of GORI caused $20 \%$ pollen germination percentage but germinated pollen has defect on tube elongation as well, causing full male sterility (Kim et al., 2021). REN4 was shown to interact with PICALM9b/EAP1 (Li et al., 2018), similarly to interactions between GORI and OsANTH3. The WD40 motif might function as a scaffold to form an endocytosis complex. Taken together, we were able to draw a working model of OsANTH in a pollen (Figure 7). We still need to further confirm how this complex affects germination signaling; however, we assume that OsANTH proteins are involved in CME by interacting with various membrane-bound proteins or signaling molecules through the C-terminal to form a functional complex.

\section{DATA AVAILABILITY STATEMENT}

The datasets presented in this study can be found in online repositories. The names of the repository/repositories and accession number(s) can be found in the article/ Supplementary Material. 


\section{AUTHOR CONTRIBUTIONS}

SL, K-HJ, and Y-JK conceived and designed the experiments, analyzed data, and wrote the manuscript. W-JH, JS, and E-JK contributed the phenotypical and computational analysis. SP contributed the research design. All authors contributed to the article and approved the submitted version.

\section{FUNDING}

This work was supported by grants from the New Breeding Center Program (PJ01492703 to K-HJ), the Rural Development Administration, Republic of Korea, the Collaborative Genome Program of the Korea Institute of Marine Science and Technology Promotion (KIMST) funded by the Ministry of

\section{REFERENCES}

Atkinson, H. A., Daniels, A., and Read, N. D. (2002). Live-cell imaging of endocytosis during conidial germination in the rice blast fungus, Magnaporthe grisea. Fungal Genet. Biol. 37, 233-244. doi: 10.1016/s1087-1845(02)00 535-2

Baluška, F., Hlavacka, A., Samaj, J., Palme, K., Robinson, D. G., Matoh, T., et al. (2002). F-actin-dependent endocytosis of cell wall pectins in meristematic root cells. Insights from brefeldin A-induced compartments. Plant Physiol. 130, 422-431. doi: 10.1104/pp.007526

Barth, M., and Holstein, S. E. (2004). Identification and functional characterization of Arabidopsis AP180, a binding partner of plant alphaC-adaptin. J. Cell Sci. 117, 2051-2062. doi: 10.1242/jcs.01062

Bolte, S., Talbot, C., Boutte, Y., Catrice, O., Read, N., and Satiat-Jeunemaitre, B. (2004). FM-dyes as experimental probes for dissecting vesicle trafficking in living plant cells. J. Microsc. 214, 159-173.

Brett, T. J., Traub, L. M., and Fremont, D. H. (2002). Accessory protein recruitment motifs in clathrin-mediated endocytosis. Structure 10, 797-809. doi: 10.1016/ s0969-2126(02)00784-0

Brod, J., Hellwig, A., and Wieland, F. T. (2020). Epsin but not AP-2 supports reconstitution of endocytic clathrin-coated vesicles. FEBS Lett. 594, 2227-2239. doi: 10.1002/1873-3468.13801

Burbea, M., Dreier, L., Dittman, J. S., Grunwald, M. E., and Kaplan, J. M. (2002). Ubiquitin and AP180 regulate the abundance of GLR-1 glutamate receptors at postsynaptic elements in C. elegans. Neuron 35, 107-120. doi: 10.1016/s08966273(02)00749-3

Cao, P., Jung, K., Choi, D., Hwang, D., Zhu, J., and Ronald, P. C. (2012). The rice oligonucleotide array database: an atlas of rice gene expression. Rice 5, 1-9. doi: 10.1186/1939-8433-5-17

Chandran, A. K. N., Hong, W., Abhijith, B., Lee, J., Kim, Y., Park, S. K., et al. (2020). Rice male gamete expression database (RMEDB): a web resource for functional genomic studies of rice male organ development. J. Plant Biol. 63, 421-430. doi: 10.1007/s12374-020-09267-1

de Craene, J., Ripp, R., Lecompte, O., Thompson, J. D., Poch, O., and Friant, S. (2012). Evolutionary analysis of the ENTH/ANTH/VHS protein superfamily reveals a coevolution between membrane trafficking and metabolism. BMC Genomics 13:297. doi: 10.1186/1471-2164-13-297

Dettmer, J., Hong-Hermesdorf, A., Stierhof, Y. D., and Schumacher, K. (2006). Vacuolar H+-ATPase activity is required for endocytic and secretory trafficking in Arabidopsis. Plant Cell 18, 715-730. doi: 10.1105/tpc.105. 037978

Dhonukshe, P., Aniento, F., Hwang, I., Robinson, D. G., Mravec, J., Stierhof, Y., et al. (2007). Clathrin-mediated constitutive endocytosis of PIN auxin efflux carriers in Arabidopsis. Curr. Biol. 17, 520-527. doi: 10.1016/j.cub.2007. 01.052

Duncan, M. C., and Payne, G. S. (2003). ENTH/ANTH domains expand to the Golgi. Trends Cell Biol. 13, 211-215. doi: 10.1016/s0962-8924(03)00 076-x
Oceans and Fisheries (MOF) (No. 20180430 to K-HJ), and the National Research Foundation (NRF), Ministry of Education, Science and Technology (NRF-2019R1C1C1002636 to Y-JK).

\section{ACKNOWLEDGMENTS}

We thank Prof. Gynheung An (Kyung Hee University) for providing T-DNA lines.

\section{SUPPLEMENTARY MATERIAL}

The Supplementary Material for this article can be found online at: https://www.frontiersin.org/articles/10.3389/fpls.2021. 609473/full\#supplementary-material

Elkin, S. R., Oswald, N. W., Reed, D. K., Mettlen, M., MacMillan, J. B., and Schmid, S. L. (2016). Ikarugamycin: a natural product inhibitor of clathrin-mediated endocytosis. Traffic 17, 1139-1149. doi: 10.1111/tra.12425

Ford, M. G., Pearse, B. M., Higgins, M. K., Vallis, Y., Owen, D. J., Gibson, A., et al. (2001). Simultaneous binding of PtdIns(4,5)P2 and clathrin by AP180 in the nucleation of clathrin lattices on membranes. Science 291, 1051-1055. doi: $10.1126 /$ science.291.5506.1051

Geldner, N., Anders, N., Wolters, H., Keicher, J., Kornberger, W., Muller, P., et al. (2003). The Arabidopsis GNOM ARF-GEF mediates endosomal recycling auxin transport, and auxin-dependent plant growth. Cell 112, 219-230. doi: 10.1016/S0092-8674(03)00003-5

Goldstein, J. L., Brown, M. S., Anderson, R. G., Russell, D. W., and Schneider W. J. (1985). Receptor-mediated endocytosis: concepts emerging from the LDL receptor system. Annu. Rev. Cell Biol. 1, 1-39. doi: 10.1146/annurev.cb.01. 110185.000245

Hasumi, K., Shinohara, C., Naganuma, S., and Endo, A. (1992). Inhibition of the uptake of oxidized low-density lipoprotein in macrophage J774 by the antibiotic ikarugamycin. Eur. J. Biochem. 205, 841-846. doi: 10.1111/j.1432-1033.1992. tb16848.x

Holstein, S. E., and Oliviusson, P. (2005). Sequence analysis of Arabidopsis thaliana E/ANTH-domain-containing proteins: membrane tethers of the clathrindependent vesicle budding machinery. Protoplasma 226, 13-21. doi: 10.1007/ s00709-005-0105-7

Hong, W., and Jung, K. (2018). Comparative analysis of flanking sequence tags of T-DNA/transposon insertional mutants and genetic variations of fast-neutron treated mutants in rice. J. Plant Biol. 61, 80-84. doi: 10.1007/s12374-0170425-0

Hong, W., Kim, Y., Chandran, A. K. N., and Jung, K. (2019). Infrastructures of systems biology that facilitate functional genomic study in rice. Rice 12:15. doi: 10.1186/s12284-019-0276-z

Hong, W., Kim, Y., Kim, E., Kumar, A. N. C., Moon, S., Gho, Y., et al. (2020). CAFRI-rice: CRISPR applicable functional redundancy inspector to accelerate functional genomics in rice. Plant J. 104, 532-545. doi: 10.1111/tpj.14926

Hurtley, S. M. (2000). Endocytosis and the nucleus. J. Cell Biol. 288:1139. doi: 10.1126/science.288.5469.1139a

Itoh, T., Koshiba, S., Kigawa, T., Kikuchi, A., Yokoyama, S., and Takenawa, T. (2001). Role of the ENTH domain in phosphatidylinositol-4, 5-bisphosphate binding and endocytosis. Science 291, 1047-1051. doi: 10.1126/science.291. 5506.1047

Jain, M., Nijhawan, A., Tyagi, A. K., and Khurana, J. P. (2006). Validation of housekeeping genes as internal control for studying gene expression in rice by quantitative real-time PCR. Biochem. Biophys. Res. Commun. 345, 646-651. doi: 10.1016/j.bbrc.2006.04.140

Kaneda, M., van Oostende-Triplet, C., Chebli, Y., Testerink, C., Bednarek, S. Y., and Geitmann, A. (2019). Plant AP180 N-terminal homolog proteins are involved in clathrin-dependent endocytosis during pollen tube growth in Arabidopsis thaliana. Plant Cell Physiol. 60, 1316-1330. doi: 10.1093/pcp/ pcz036 
Kim, E., Park, S., Hong, W., Silva, J., Liang, W., Zhang, D., et al. (2020). Genomewide analysis of RopGEF gene family to identify genes contributing to pollen tube growth in rice (Oryza sativa). BMC Plant Biol. 20:95. doi: 10.1186/s12870020-2298-5

Kim, E. J., Kim, Y. J., Hong, W. J., Lee, C., Jeon, J. S., and Jung, K. H. (2019). Genome-wide analysis of root hair preferred $\mathrm{RBOH}$ genes suggests that three $\mathrm{RBOH}$ genes are associated with auxin-mediated root hair development in rice. J. Plant Biol. 62, 229-238.

Kim, Y., Jeong, H. Y., Kang, S., Silva, J., Kim, E., Park, S. K., et al. (2020). Physiological importance of pectin modifying genes during rice pollen development. Int. J. Mol. 21:4840. doi: 10.3390/ijms21144840

Kim, Y., Kim, M., Hong, W., Moon, S., Kim, E., Silva, J., et al. (2021). GORI encoding the WD40 domain protein is required for pollen tube germination and elongation in rice. Plant J. 105, 1645-1664. doi: 10.1111/tpj.15139

Kleine-Vehn, J., Dhonukshe, P., Swarup, R., Bennett, M., and Friml, J. (2006). Subcellular trafficking of the Arabidopsis auxin influx carrier AUX1 uses a novel pathway distinct from PIN1. Plant Cell 18, 3171-3181. doi: 10.1105/tpc.106. 042770

Lacy, M. M., Ma, R., Ravindra, N. G., and Berro, J. (2018). Molecular mechanisms of force production in clathrin-mediated endocytosis. FEBS Lett. 592, 35863605. doi: 10.1002/1873-3468.13192

Lam, S. K., Cai, Y., Tse, Y. C., Wang, J., Law, A. H. Y., Pimpl, P., et al. (2009). BFA-induced compartments from the Golgi apparatus and trans-Golgi network/early endosome are distinct in plant cells. Plant J. 60, 865-881. doi: 10.1111/j.1365-313x.2009.04007.x

Lam, S. K., Siu, C. L., Hillmer, S., Jang, S., An, G., Robinson, D. G., et al. (2007). Rice SCAMP1 defines clathrin-coated, trans-golgi-located tubular-vesicular structures as an early endosome in tobacco BY-2 cells. Plant Cell 19, 296-319. doi: $10.1105 /$ tpc.106.045708

Legendre-Guillemin, V., Wasiak, S., Hussain, N. K., Angers, A., and McPherson, P. S. (2004). ENTH/ANTH proteins and clathrin-mediated membrane budding. J. Cell Sci. 117(Pt 1), 9-18. doi: 10.1242/jcs.00928

Li, H., Luo, N., Wang, W., Liu, Z., Chen, J., Zhao, L., et al. (2018). The REN4 rheostat dynamically coordinates the apical and lateral domains of Arabidopsis pollen tubes. Nat. Commun. 9:2573. doi: 10.1038/s41467-018-04838-w

Lippincott-Schwartz, J., Yuan, L., Tipper, C., Amherdt, M., Orci, L., and Klausner, R. D. (1991). Brefeldin A's effects on endosomes, lysosomes, and the TGN suggest a general mechanism for regulating organelle structure and membrane traffic. Cell 67, 601-616. doi: 10.1016/0092-8674(91)90534-6

Luo, T., Fredericksen, B. L., Hasumi, K., Endo, A., and Garcia, J. V. (2001). Human immunodeficiency virus type 1 Nef-induced CD4 cell surface downregulation is inhibited by ikarugamycin. J. Virol. 75, 2488-2492. doi: 10.1128/JVI.75.5.24882492.2001

Metzler, M., Gan, L., Wong, T. P., Liu, L., Helm, J., Liu, L., et al. (2007). NMDA receptor function and NMDA receptor-dependent phosphorylation of huntingtin is altered by the endocytic protein HIP1. J. Neurosci. 27, 2298-2308. doi: 10.1523/JNEUROSCI.5175-06.2007

Miller, W. E., and Lefkowitz, R. J. (2001). Arrestins as signaling molecules involved in apoptotic pathways: a real eye opener. Sci. STKE 2001:e1. doi: 10.1126/stke. 2001.69.pe1

Mishra, S. K., Keyel, P. A., Hawryluk, M. J., Agostinelli, N. R., Watkins, S. C., and Traub, L. M. (2002). Disabled-2 exhibits the properties of a cargo-selective endocytic clathrin adaptor. EMBO J. 21, 4915-4926. doi: 10.1093/emboj/cdf487

Moon, S., Chandran, A. K. N., Kim, Y. J., Gho, Y., Hong, W. J., An, G., et al. (2019). Rice RHC encoding a putative cellulase is essential for normal root hair elongation. J. Plant Biol. 62, 82-91.

Moscatelli, A. (2008). Endocytic pathways in pollen tube: implications for in vivo growth regulation. Plant Signal. Behav. 3, 325-327. doi: 10.4161/psb.3.5. 5340

Moscatelli, A., Ciampolini, F., Rodighiero, S., Onelli, E., Cresti, M., Santo, N., et al. (2007). Distinct endocytic pathways identified in tobacco pollen tubes using charged nanogold. J. Cell Sci. 120, 3804-3819. doi: 10.1242/jcs.012138

Muro, K., Matsuura-Tokita, K., Tsukamoto, R., Kanaoka, M. M., Ebine, K., Higashiyama, T., et al. (2018). ANTH domain-containing proteins are required for the pollen tube plasma membrane integrity via recycling ANXUR kinases. Commun. Biol. 1:152. doi: 10.1038/s42003-018-0158-8

Nagawa, S., Xu, T., Lin, D., Dhonukshe, P., Zhang, X., Friml, J., et al. (2012). ROP GTPase-dependent actin microfilaments promote PIN1 polarization by localized inhibition of clathrin-dependent endocytosis. PLoS Biol. 10:e1001299. doi: 10.1371/journal.pbio.1001299

Naito, Y., Hino, K., Bono, H., and Ui-Tei, K. (2015). CRISPRdirect: software for designing CRISPR/Cas guide RNA with reduced off-target sites. Bioinformatics 31, 1120-1123. doi: 10.1093/bioinformatics/btu743

Nebenführ, A., Ritzenthaler, C., and Robinson, D. G. (2002). Brefeldin A: deciphering an enigmatic inhibitor of secretion. Plant Physiol. 130, 1102-1108. doi: 10.1104/pp.011569

Nguyen, H. H., Lee, M. H., Song, K., Ahn, G., Lee, J., and Hwang, I. (2018). The A/ENTH domain-containing protein AtECA4 is an adaptor protein involved in cargo recycling from the trans-Golgi network/early endosome to the plasma membrane. Mol. Plant 11, 568-583. doi: 10.1016/j.molp.2018.01.001

Nguyen, Q., Lee, Y., Cho, L., Jeong, H., An, G., and Jung, K. (2015). Genomewide identification and analysis of Catharanthus roseus RLK1-like kinases in rice. Planta 241, 603-613. doi: 10.1007/s00425-014-2203-2

Owen, D. J., Vallis, Y., Noble, M. E., Hunter, J. B., Dafforn, T. R., Evans, P. R., et al. (1999). A structural explanation for the binding of multiple ligands by the $\alpha$-adaptin appendage domain. Cell 97, 805-815.

Pagnussat, L., Burbach, C., Baluška, F., and de la Canal, L. (2012). An extracellular lipid transfer protein is relocalized intracellularly during seed germination. J. Exp. Bot. 63, 6555-6563. doi: 10.1093/jxb/ers311

Pfaffl, M. (2001). A new mathematical model for relative quantification in real-time RT-PCR. Nucleic Acids Res. 29:e45. doi: 10.1093/nar/29.9.e45

Prasad, K., and Lippoldt, R. E. (1988). Molecular characterization of the AP180 coated vesicle assembly protein. Biochemistry 27, 6098-6104. doi: 10.1021/ bi00416a040

Reynolds, G. D., Wang, C., Pan, J., and Bednarek, S. Y. (2018). Inroads into internalization: five years of endocytic exploration. Plant Physiol. 176, 208-218. doi: $10.1104 / p p .17 .01117$

Robinson, D. G., Jiang, L., and Schumacher, K. (2008). The endosomal system of plants: charting new and familiar territories. Plant Physiol. 147, 1482-1492. doi: 10.1104/pp.108.120105

Rosquete, M. R., Davis, D. J., and Drakakaki, G. (2018). The plant trans-golgi network: not just a matter of distinction. Plant Physiol. 176, 187-198. doi: 10.1104/pp.17.01239

Samaj, J., Baluska, F., Voigt, B., Schlicht, M., and Menzel, D. (2004). Endocytosis, actin cytoskeleton, and signaling. Plant Physiol. 135, 1150-1161. doi: 10.1104/ pp. 104.040683

Scheele, U., Kalthoff, C., and Ungewickell, E. (2001). Multiple interactions of auxilin 1 with clathrin and the AP-2 adaptor complex. J. Biol. Chem. 276, 36131-36138. doi: 10.1074/jbc.M106511200

Silver, N., Best, S., Jiang, J., and Thein, S. L. (2006). Selection of housekeeping genes for gene expression studies in human reticulocytes using real-time PCR. BMC Mol. Biol. 7:33. doi: 10.1186/1471-2199-7-33

Skruzny, M., Desfosses, A., Prinz, S., Dodonova, S. O., Gieras, A., Uetrecht, C., et al. (2015). An organized co-assembly of clathrin adaptors is essential for endocytosis. Dev. Cell 33, 150-162. doi: 10.1016/j.devcel.2015.02.023

Song, K., Jang, M., Kim, S. Y., Lee, G., Lee, G. J., Kim, D. H., et al. (2012). An A/ENTH domain-containing protein functions as an adaptor for clathrincoated vesicles on the growing cell plate in Arabidopsis root cells. Plant Physiol. 159, 1013-1025. doi: 10.1104/pp.112.199380

Vo, K. T. X., Kim, C., Chandran, A. K. N., Jung, K., An, G., and Jeon, J. (2015). Molecular insights into the function of ankyrin proteins in plants. J. Plant Biol. 58, 271-284. doi: 10.1007/s12374-015-0228-0

Wang, H., Huang, J., and Jauh, G. (2010). Pollen germination and tube growth. Adv. Bot. Res. 54, 1-52. doi: 10.1016/S0065-2296(10)54001-1

Wang, Q., Kong, L., Hao, H., Wang, X., Lin, J., Samaj, J., et al. (2005). Effects of brefeldin A on pollen germination and tube growth. Antagonistic effects on endocytosis and secretion. Plant Physiol. 139, 1692-1703. doi: 10.1104/pp.105. 069765

Wendland, B., Steece, K. E., and Emr, S. D. (1999). Yeast epsins contain an essential $\mathrm{N}$-terminal ENTH domain, bind clathrin and are required for endocytosis. EMBO J. 18, 4383-4393. doi: 10.1093/emboj/18.16.4383

Yao, P. J., Coleman, P. D., and Calkins, D. J. (2002). High-resolution localization of clathrin assembly protein AP180 in the presynaptic terminals of mammalian neurons. J. Comp. Neurol. 447, 152-162. doi: 10.1002/cne.10217

Zhao, Y., Yan, A., Feijo, J. A., Furutani, M., Takenawa, T., Hwang, I., et al. (2010). Phosphoinositides regulate clathrin-dependent endocytosis at the tip of pollen 
tubes in Arabidopsis and tobacco. Plant Cell 22, 4031-4044. doi: 10.1105/tpc. 110.076760

Zimmermann, P., Hirsch-Hoffmann, M., Hennig, L., and Gruissem, W. (2004). GENEVESTIGATOR. Arabidopsis microarray database and analysis toolbox. Plant Physiol. 136, 2621-2632. doi: 10.1104/pp.104.04 6367

Zouhar, J., and Sauer, M. (2014). Helping hands for budding prospects: ENTH/ANTH/VHS accessory proteins in endocytosis, vacuolar transport, and secretion. Plant Cell 26, 4232-4244. doi: 10.1105/tpc.114.13 1680
Conflict of Interest: The authors declare that the research was conducted in the absence of any commercial or financial relationships that could be construed as a potential conflict of interest.

Copyright (c) 2021 Lee, Hong, Silva, Kim, Park, Jung and Kim. This is an open-access article distributed under the terms of the Creative Commons Attribution License (CC BY). The use, distribution or reproduction in other forums is permitted, provided the original author(s) and the copyright owner(s) are credited and that the original publication in this journal is cited, in accordance with accepted academic practice. No use, distribution or reproduction is permitted which does not comply with these terms. 